\title{
INFLUENCIA DE ESTRATEGIAS DOCENTES Y METODOLOGÍAS EDUCATIVAS EN EL APRENDIZAJE SIGNIFICATIVO DE ESTUDIANTES DE TERAPIA OCUPACIONAL
}

\author{
THE INFLUENCE OF TEACHING TEACHING STRATEGIES AND TEACHING \\ METHODOLOGIES ON MEANINGFUL LEARNING OF \\ OCCUPATIONAL THERAPY STUDENTS
}

\section{Julie Vásquez Duque¹, Lisette González Duk²}

\begin{abstract}
RESUMEN
La presente investigación tuvo como objetivo identificar la influencia de estrategias docentes y metodologías educativas en la adquisición del aprendizaje significativo de estudiantes de la carrera de Terapia Ocupacional de una universidad privada de Santiago, entendiendo metodologías educativas como la forma y recursos utilizados para presentar el contenido y como estrategias las acciones del docente orientadas a favorecer la adquisición del aprendizaje.

Para ello se consideraron como objetivos específicos: identificar la frecuencia con que se aplican las estrategias y metodologías por los docentes; identificar el grado en que las estrategias y metodologías influyen en la disposición de los estudiantes hacia el aprendizaje; e identificar estrategias y metodologías específicas que los estudiantes relacionan con el desarrollo de su aprendizaje significativo.

Se elaboró una encuesta en línea de 9 preguntas con escala tipo Likert y los resultados obtenidos indicaron que existe alta influencia de las metodologías y estrategias utilizadas en el aprendizaje significativo.
\end{abstract}

\section{PALABRAS CLAVE}

Estrategias docentes, metodologías docentes, aprendizaje significativo, terapia ocupacional.

1 Terapeuta Ocupacional, Magister en Educación en Ciencias de la Salud. Profesora asistente, Escuela de Terapia Ocupacional. Universidad San Sebastián. ORCID iD 0000-0002-0295-0072.

2 Tecnólogo Médico, Magíster en Educación en Ciencias de la Salud. Docente Universidad San Sebastián. ORCID iD 0000-0002-4253-4911. 


\section{ABSTRACT}

The goal of this research was to identify the influence of teaching strategies and methodologies on significant learning of Occupational Therapy students in a private university at Santiago, understanding teaching methodologies as the way and resources used to introduce the content, and strategies as the actions oriented towards benefiting learning acquisition.

With this aim, the authors considered as specific objectives to identify the frequency with which professors use strategies and methodologies; identify the magnitude of these strategies and methodologies on the student's disposition towards learning; and identify specific strategies and methodologies that students relate to the development of their effective learning.

The authors elaborated an online survey with 9 questions with a Likert type scale. The results show high influence of methodologies and strategies on significant learning.

\section{KEYWORDS}

Teaching strategies and methodologies, significant learning, occupational therapy.

Recibido: 30/05/2017

Aceptado: 16/11/2017 


\section{INTRODUCCIÓN}

A raíz del proceso de acreditación de Escuela de Terapia Ocupacional de una universidad privada de Santiago y la ecualización de programas de sus sedes, se requiere revisar la metodología de enseñanza que se ha utilizado y los resultados de aprendizaje obtenidos hasta el momento.

Es por esto que surge la necesidad de conocer la opinión de los estudiantes respecto de las metodologías educativas y estrategias docentes de las que han sido partícipes, y su influencia en su aprendizaje.

De acuerdo a Ausubel (1953), citado por Palmero (2008), el aprendizaje significativo corresponde a un proceso multifactorial, que considera los elementos y condiciones que garantizan la adquisición, la asimilación y la retención de nuevos contenidos que tienen sentido y significado para quien está aprendiendo. Esta teoría expone que para que se produzca aprendizaje significativo deben darse dos condiciones fundamentales; la primera relacionada con el estudiante, quien debe tener disposición para aprender de manera significativa, la segunda condición corresponde a la presentación del material potencialmente significativo.

Esta teoría pretende determinar las maneras adecuadas y más eficientes de organizar y presentar materiales de estudio, con el objetivo de motivar y dirigir deliberadamente el aprendizaje hacia metas concretas.

El presente estudio considera metodologías educativas de acuerdo a la definición de Díaz (2005), como: un conjunto de decisiones sobre los procedimientos a emprendery sobre los recursos a utilizar en las diferentes fases de un plan de acción. De acuerdo a este autor, estos procedimientos y recursos deben ser organizados y secuenciados coherentemente con los resultados de aprendizaje en cada momento del proceso, la que debe adecuarse a las características y situación real del estudiante, considerando su desarrollo cognitivo y promoviendo que aprenda significativamente.

Las metodologías educativas abordadas en el siguiente estudio corresponden a la clasificación de Jenaro, Flores et al, (2013) en su investigación sobre "Metodologías docentes en la educación superior: percepciones del profesorado sobre su importancia y uso". Estas son: Clase magistral, debate, eventos científicos, trabajos autónomos, seminarios de investigación, estudios de casos, prácticas externas, prácticas en laboratorio, presentaciones orales y resolución de problemas.

Como estrategia docente de enseñanza o aprendizaje, se consideran procedimientos flexibles y adaptativos a distintas circunstancias de enseñanza. Según la clasificación de Díaz \& Hernández (1999) se pueden agrupar en:

- Estrategias para activar (o generar) conocimientos previos y para establecer expectativas adecuadas en los alumnos.

- Estrategias para orientar la atención de los alumnos.

- Estrategias para organizar la información que se ha de aprender.

- Estrategias para promover el enlace entre los conocimientos previos y la nueva información que se ha de aprender.

Respecto del estado del arte, Rodríguez \& González (2013) realizan un estudio sobre la percepción de los estudiantes de Odontología de las estrategias metodológicas más satisfactorias utilizadas por sus académicos, encontrando que las tres formas identificadas por los alumnos como más satisfactorias y significativas para el aprendizaje en aula fueron: Aprendizaje Basado en Problemas, Análisis y Presentación de Casos Clínicos y Clase expositiva.

Por otro lado, en el área en Terapia Ocupacional, destacan el estudio titulado "Evaluación de estrategias docentes utilizadas por profesores para la adquisición de competencias en Terapia Ocupacional", realizado en la Universidad de Salamanca el año 2010 por Flores, N., Jenaro, C., Caballo, C., Ortiz, V. \& García-Pérez, C., el que indica que las metodologías docentes más utilizadas son la clase magistral, presentaciones/exposiciones y trabajos autónomos y las menos utilizadas metodologías integradoras o aprendizaje basado en problemas. Mencionando que la percepción de los estudiantes respecto de la eficacia de estas metodologías es completamente contraria a su frecuencia de utilización.

En Chile, Farías \& López el año 2013 investigaron respecto de las necesidades de los estudiantes en su proceso de formación, en el que los estudiantes manifiestan la necesidad poner en práctica lo aprendido y recalcan la importancia de la relación que se establece entre el docente y los estudiantes. 
En este proceso, surge la interrogante ¿̇en qué grado consideran los estudiantes de la carrera, que las metodologías educativas y estrategias docentes propician las condiciones fundamentales, para la adquisición del aprendizaje significativo?

Para dilucidar esta inquietud se realiza un estudio cuantitativo de tipo exploratorio a través de una encuesta a los estudiantes. Se plantea como objetivo general "identificar la influencia de estrategias y metodologías docentes en la adquisición de aprendizaje significativo, según la opinión de estudiantes de la Carrera Terapia Ocupacional de la Universidad San Sebastián Sede Santiago", para lo que se obtiene información respecto de las estrategias y metodologías que los estudiantes consideran más utilizadas por los docentes, cuáles consideran que influyen positivamente en su disposición hacia el aprendizaje y cuáles relacionan directamente con el desarrollo de su aprendizaje.

\section{MATERIAl y MÉTODO}

Para el logro de los objetivos se plantea una investigación de tipo cuantitativa, transversal exploratoriodescriptivo, de acuerdo a la clasificación de Hernández, Fernández \& Baptista (2010), la que se llevó a cabo a través de una encuesta creada específicamente para el estudio y validada por opinión de 3 expertos. La encuesta consiste en 8 preguntas; las dos primeras destinadas a la identificación del estudiante según año de ingreso y curso actual y las preguntas posteriores dirigidas a conocer la opinión de los estudiantes respecto de la frecuencia de metodologías y estrategias utilizadas por los docentes, el grado de motivación que les genera y grado en que la presentación del material permite la relación de conocimientos nuevos con previos.

Se convocó a todos los estudiantes que se encontraban cursando Terapia Ocupacional entre tercer y décimo semestre a través de un correo electrónico en el que se les explicaban los objetivos de la investigación y la importancia de conocer su opinión para la mejora continua de la carrera, lo que conduce a un beneficio para los mismos estudiantes. Posteriormente, se envió un enlace para responder la encuesta en línea. Finalmente, la muestra total estuvo conformada por 92 estudiantes, de los cuales alrededor del $15 \%$ tuvo dificultad para responder la encuesta en línea, y manifestaron su interés por participar respondiendo la encuesta en forma impresa.

\section{Resultados}

Para identificar la influencia de estrategias y metodologías docentes en la adquisición aprendizaje significativo se presentan los resultados agrupándolos por metodologías y estrategias.

Los estudiantes señalan haber sido partícipes de todas las metodologías evaluadas, de las cuales consideran más utilizadas las prácticas en laboratorio (79\%), resolución de problemas o ejercicios en el aula ( $77 \%)$, presentaciones de estudiantes $(76 \%)$, trabajos autónomos (73\%) y estudios de casos (70\%), seguido por prácticas externas $(67 \%)$ y clase magistral (54\%). Las metodologías docentes que los estudiantes consideran menos utilizadas son seminarios de investigación (35\%), debate ( $14 \%$ ) y eventos científicos (12\%).

Respecto de las estrategias evaluadas, los estudiantes indican haber presenciado la aplicación de todas ellas por parte de sus docentes, de las cuales el dar ejemplos o analogías sería la más frecuente $(78 \%)$, seguida por mostrar ilustraciones, imágenes o esquemas que clarifican los contenidos de la clase (72\%); realizar preguntas en distintos momentos que estimulan la reflexión $(66 \%)$ y mencionar puntos claves a considerar sobre el tema (63\%). Las estrategias pre instruccionales y post instruccionales, que apuntan a la organización del conocimiento, serían las menos utilizadas, correspondiendo el (47\%) a dar a conocer los objetivos de la clase, (29\%) a utilizar mapas conceptuales para graficar los conceptos, $(29 \%)$ realizar resumen de los conceptos abordados en clase y (28\%) a dar a conocer la secuencia que tendrá la clase.

Al analizar la frecuencia de utilización de las metodologías y estrategias docentes por año de ingreso a la carrera, se observa la similitud en el patrón de respuesta. Se infiere que la diferencia en cuanto a metodologías puede estar dada por el nivel de formación académica en que se encuentran, ya que quienes ingresaron el 2010, en su mayoría se encuentran egresados o realizando la práctica profesional, por lo que han tenido mayor contacto con usuarios/pacientes y el estudio de sus casos clínicos. Considerando que la mayoría de quienes ingresaron el año 2012 se deberían encontrar en $4^{\circ}$ año de carrera, se explica que identifiquen con mayor frecuencia las prácticas externas, ya que es el año en que se realizan las pre prácticas profesionales. 


\section{GRÁFICO 1}

INFLUENCIAS DE METODOLOGÍAS DOCENTES EN EL APENDIZAJE SIGNIFICATIVO DE LOS ESTUDIANTES DE TERAPIA OCUPACIONAL DE UNA UNIVERSIDAD PRIVADA DE SANTIAGO, 2015

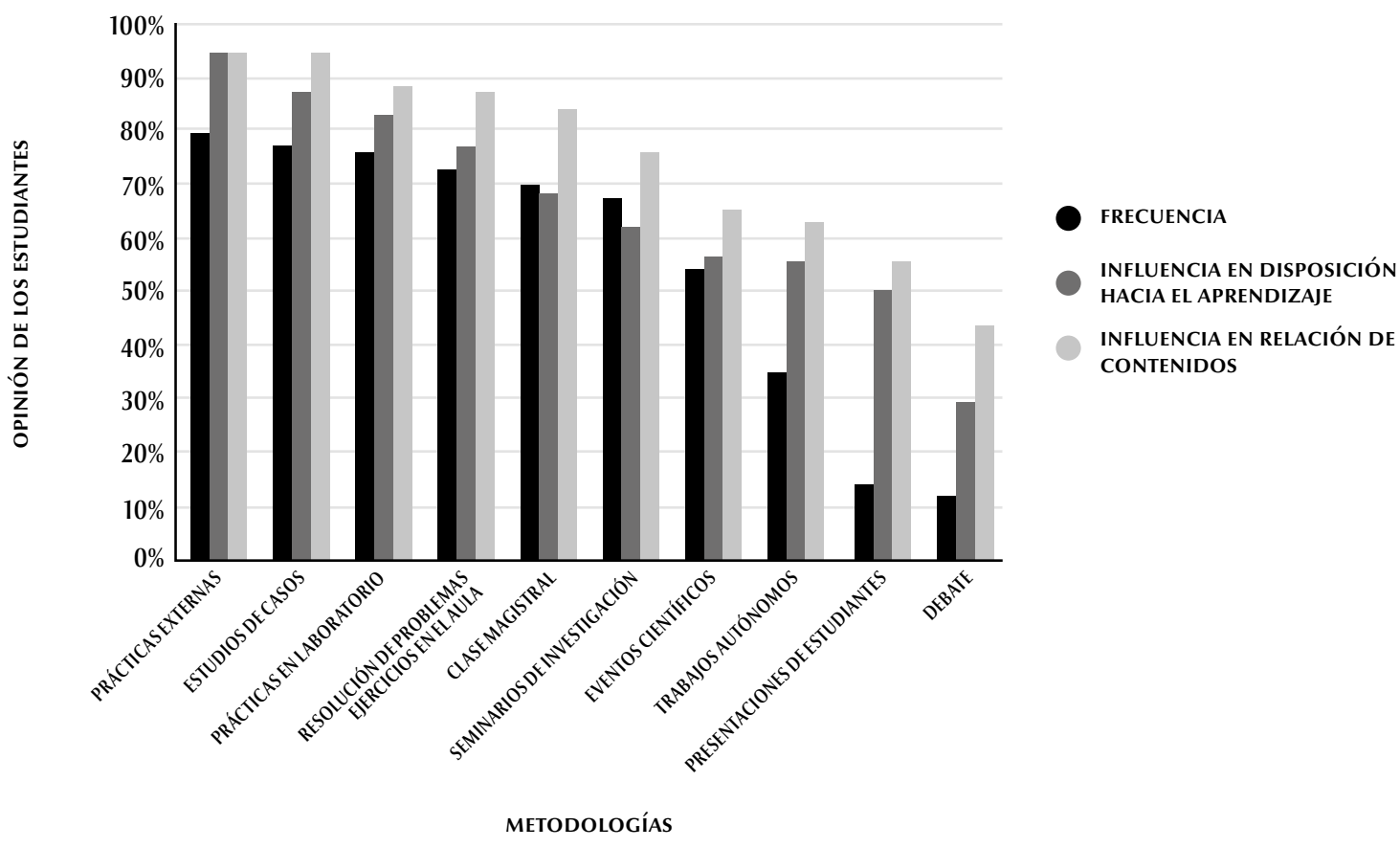

\section{GRÁFICO 2}

INFLUENCIAS DE ESTRATEGIAS DOCENTES EN EL APENDIZAJE SIGNIFICATIVO DE LOS ESTUDIANTES DE TERAPIA OCUPACIONAL UNIVERSIDAD SAN SEBASTIÁN, SEDE SANTIAGO, 2015

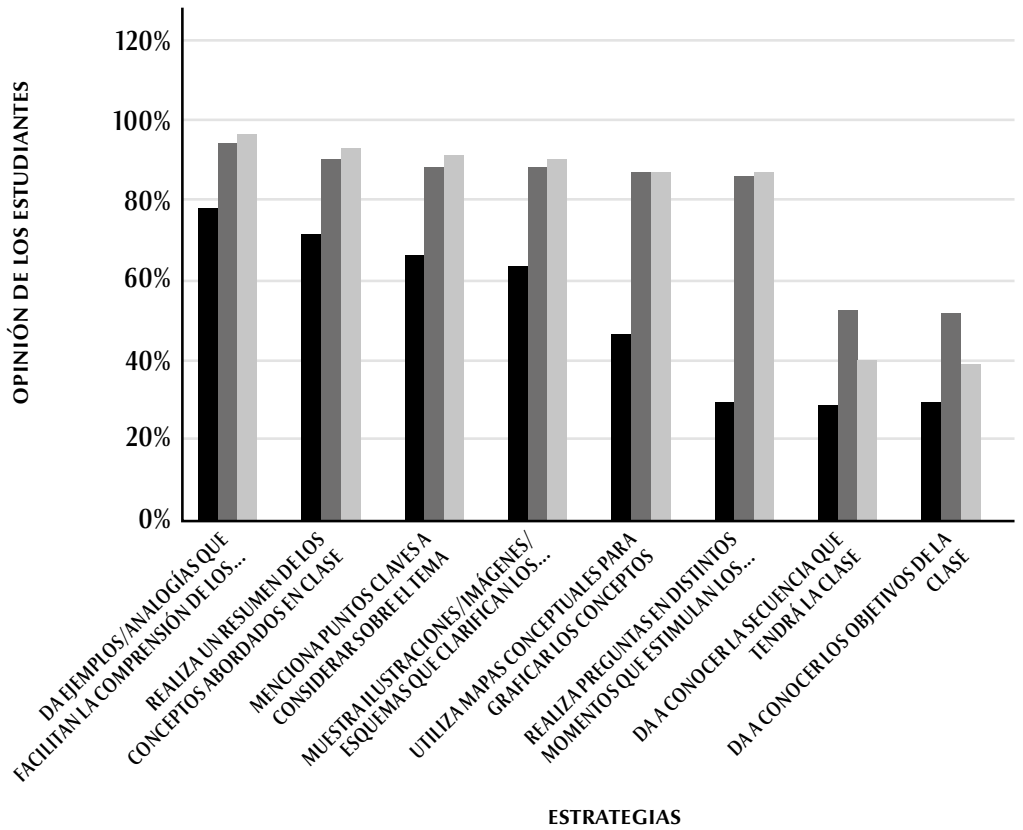

FRECUENCIA

INFLUENCIA EN DISPOSICIÓN HACIA EL APRENDIZAJE

INFLUENCIA EN RELACIÓN DE CONTENIDOS 
GRÁFICO 3

PORCENTAJE DE ESTUDIANTES QUE CONSIDERAN FRECUENCIA DE UTILIZACIÓN DE METODOLOGÍAS "SIEMPRE" Y "LA MAYORÍA DE LAS VECES" POR AÑO DE INGRESO DE ESTUDIANTES DE TERAPIA OCUPACIONAL DE UNA UNIVERSIDAD PRIVADA DE SANTIAGO, AÑO 2015

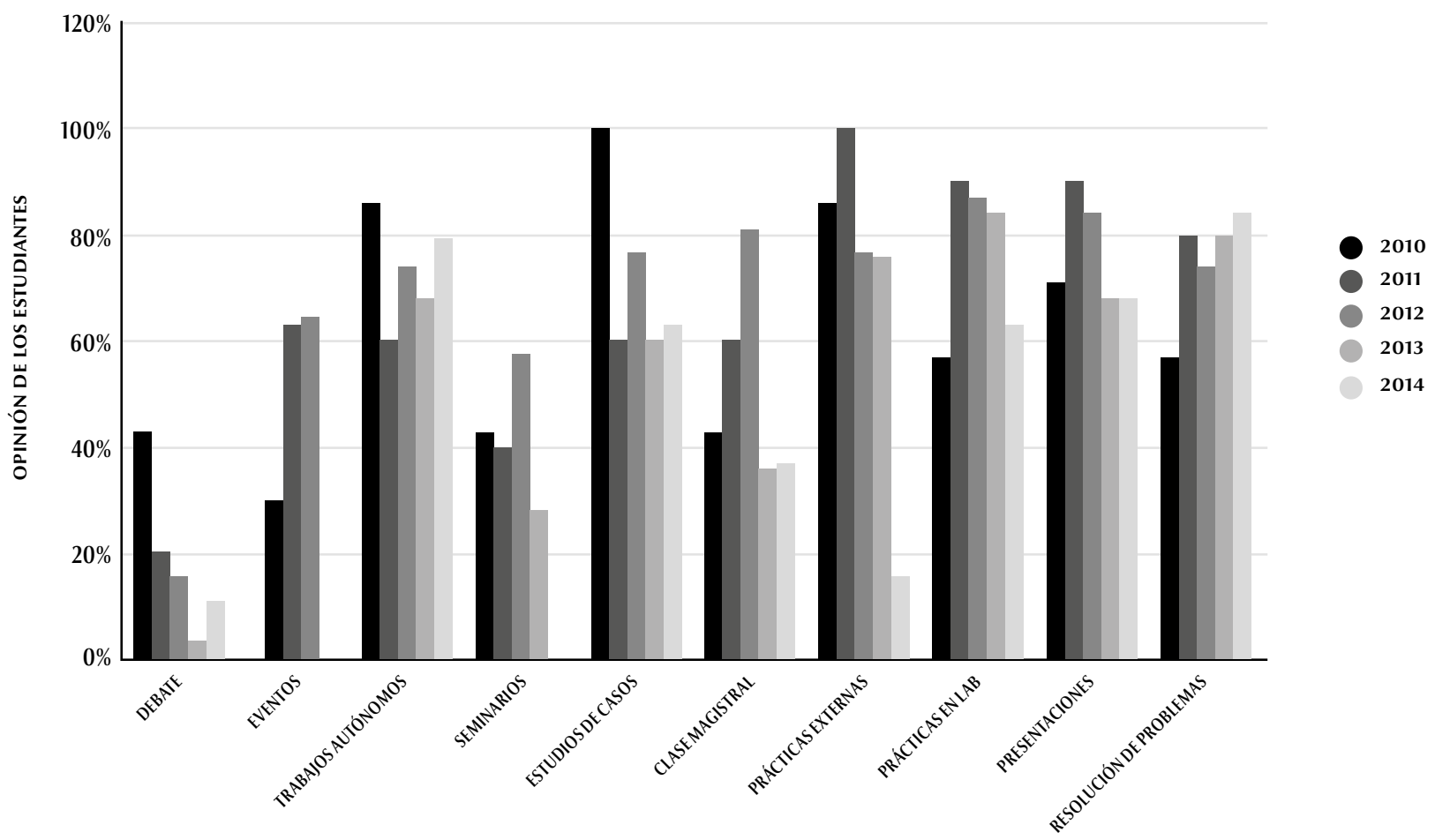

En relación al grado de motivación que generan las metodologías educativas evaluadas, los estudiantes consideran que las prácticas externas, estudios de casos, prácticas en laboratorio y resolución de problemas son las más motivantes, correspondiendo todas ellas a metodologías que demandan la participación activa por parte del estudiante, recibiendo apoyo permanente del docente. Las metodologías que menos motivación generan corresponden a aquellas que demandan mayor nivel de autonomía por parte del estudiante, como son debate, presentaciones de estudiantes y trabajos autónomos.

Respecto de estrategias, los estudiantes identifican la mayoría de ellas como altamente motivantes, excluyendo las estrategias orientadas a dar estructura, como son dar a conocer los objetivos de la clase y la secuencia que tendrá.

Al comparar los resultados de las preguntas, se observa estrecha relación entre la influencia de las estrategias docentes en la disposición hacia el aprendizaje y en la relación de contenidos nuevos con los adquiridos previamente, no así con la frecuencia de utilización. Si bien los estudiantes identifican como acciones que los docentes llevan a cabo, la frecuencia no está relacionada necesariamente con el nivel de influencia.

\section{DisCUSIÓN Y CONCLUSIONES}

Se concluye que los estudiantes de la Carrera de Terapia Ocupacional de esta universidad conocen las metodologías y estrategias utilizadas, y consideran que en su formación se utilizan frecuentemente una amplia gama de metodologías y estrategias docentes; sin embargo, existen estrategias co-instruccionales poco utilizadas que son identificadas por los estudiantes como altamente significativas, como mostrar ilustraciones/ imágenes/esquemas que clarifican los contenidos de la clase, utilizar mapas conceptuales para graficar los conceptos, y realizar preguntas en distintos momentos que estimulen la reflexión. 
Llama la atención que estrategias pre instruccionales y post instruccionales que contribuyen a la organización y estructura del conocimiento están relacionadas con menor frecuencia de utilización por parte de los docentes; esto podría indicar que la menor influencia se debe a un grado menor de habituación a este tipo de estrategia y sería interesante de investigar en mayor profundidad en estudios posteriores, ya que estas estrategias serían de gran ayuda en el estudio individual para el desarrollo del razonamiento terapéutico que se debe realizar en cualquier intervención de Terapia Ocupacional.

Los estudiantes de la Escuela de Terapia Ocupacional de esta universidad consideran que un alto porcentaje de las estrategias y metodologías docentes utilizadas en su formación contribuyen a que se presenten las condiciones necesarias para que se produzca el aprendizaje significativo; indicando como principales metodologías y estrategias docentes que influyen positivamente en su disposición al aprendizaje aquellas que se relacionan con la participación activa en el desarrollo de la clase, en la que deba desarrollar el razonamiento terapéutico y desplegar habilidades y destrezas para la obtención de información, análisis y propuesta de soluciones en una relación colaborativa con terceros. Esto se relaciona directamente con la teoría de Ausubel creada en 1963, ya que la participación activa motiva una disposición hacia el aprendizaje significativo y demanda que se evoquen aprendizajes y contenidos previos para dar una respuesta.

Respecto de la evidencia bibliográfica nacional e internacional revisada para el presente estudio, los resultados coinciden con la investigación realizada en la carrera de Odontología el año 2013, pero difieren de los antecedentes registrados en la literatura específica de Terapia Ocupacional. Si recordamos, tanto el estudio realizado en España por Flores, N., Jenaro, C., Caballo, C., Ortiz, V. \& García-Pérez, C. (2010) y el realizado en Chile por Farías \& López (2013), ambas indican que la metodología docente más utilizada en la carrera de Terapia Ocupacional es la clase magistral, mientras que los estudiantes consideran necesaria la incorporación de metodologías activas que fomenten la autonomía y proactividad. Señalan, además, que las metodologías de mayor utilidad corresponden a la resolución de problemas y ejercicios en el aula, realización de prácticas externas y debate. Las metodologías de menor utilidad según los estudiantes serían la clase magistral y trabajos autónomos.
Por otro lado, la presente investigación señala que los docentes de esta carrera utilizan frecuentemente metodologías que demandan la participación activa de los estudiantes, las que son consideradas por los estudiantes motivantes e influyentes en su aprendizaje. Por ejemplo: prácticas de laboratorio, resolución de problemas o ejercicios en el aula y presentaciones de estudiantes.

La presente investigación confirma que las metodologías que influyen en mayor grado son aquellas que demandan la participación activa por parte del estudiante: prácticas externas, estudios de casos, prácticas en laboratorio y resolución de problemas o ejercicios en el aula; sin embargo hay discrepancia en relación a debate, el que en la presente investigación es identificado como una de las metodologías menos influyentes, junto con trabajos autónomos y presentaciones de estudiantes.

Es importante destacar que las metodologías identificadas por los estudiantes como más influyentes corresponden a las que son guiadas por los docentes, donde existen expectativas explícitas en relación al desempeño de los estudiantes, quienes se adaptan a ellas. Se infiere que esto otorga mayor seguridad. Por otro lado, el que metodologías que demanden mayor autonomía sean consideradas menos motivantes e influyentes resulta preocupante, ya que son justamente éstas las que reflejan la capacidad de aprender a aprender, lo que es imprescindible en profesionales del área de la salud, de quienes se espera que en un futuro contribuyan a la creación de nuevo conocimiento.

Estos resultados también podrían indicar baja valoración de lo teórico y cognitivo, lo que sería interesante de analizar en relación al desempeño de los estudiantes en estudios posteriores, sobre todo considerando que Terapia Ocupacional es una profesión universitaria, en la que por definición es fundamental el desarrollo del conocimiento y enriquecimiento desde la teoría a la práctica y viceversa.

A partir de esto surge la interrogante ¿están aprendiendo los estudiantes en forma significativa real, o valoran un aprendizaje que les permita cumplir con las expectativas del docente para aprobar la asignatura? 


\section{REFERENCIAS BibLIOGRÁFICAS}

De Miguel Díaz, Mario; Alfaro Rocher, I. J.; Apodaca Urquijo, P.; Arias Blanco, J.M.; García Jiménez, E.; Lobato Fraile, C. \& Pérez Boullosa, A. (2005). Modalidades de enseñanza centradas en el desarrollo de competencias: Orientaciones para promover el cambio metodológico en el espacio europeo de educación superior. España: Universidad de Oviedo.

Díaz Barriga Arceo, F. \& Hernández Rojas, G. (2002). Estrategias docentes para un aprendizaje significativo: Una interpretación constructivista ( $2^{\text {da }}$ ed.). México: McGraw-Hill/Interamericana.

Farías, L. \& López, C. (2013). La formación de pregrado de Terapia Ocupacional en Chile vista desde la perspectiva de los estudiantes: ¿Cuál es la percepción de necesidades que tienen los estudiantes de Terapia Ocupacional en relación a su proceso de formación? Revista Chilena de Terapia Ocupacional, 13(1), 43-50.

Flores Robaina N.E., Jenaro Río, C. Caballo Escribano, C., García-Pérez, M.C. \& Ortiz Oria, V. (2012). Adquisición de competencias en el grado de Terapia Ocupacional, a través del desarrollo de estrategias docentes en el profesorado de la titulación. Memoria ID11-007. Ayudas de la Universidad de Salamanca para la innovación docente, curso 2011-2012. Informe Final de Ejecución.

Flores, N., Jenaro, C., Caballo, C., Ortiz, V. \& García-Pérez, C. (2010). Evaluación de estrategias docentes utilizadas por profesores para la adquisición de competencias en Terapia Ocupacional. Comunicación presentada en las X Jornadas de Redes de Investigación sobre Docencia Universitaria celebradas en Alicante los días 7 y 8 de junio de 2012. España.

Hernández Sampieri, Roberto, Fernández Collado, Carlos \& Baptista Lucio, Pilar. (2010). Metodología de la investigación ( $5^{\mathrm{a}}$ ed.) México: McGraw-Hill.

Jenaro, C., Flores, N., Poy, R., González-Gil, F. \& Martín-Pastor, E. (2013). Metodologías docentes en la educación superior: Percepciones del profesorado sobre su importancia y uso. Revista de Enseñanza Universitaria, 39(1), 1-16.

Latorre Ariño, M. \& Seco del Pozo, C. (2013). Metodología: Estrategias y Técnicas metodológica (1 ${ }^{\mathrm{a}}$ ed, ) Lima: Perú.

Rodríguez Palmero, M. (2008). La teoría del aprendizaje significativo en la perspectiva de la psicología cognitiva $\left(1^{\mathrm{a}}\right.$ ed) Barcelona: Octaedro. Rodríguez, M. P. \& González, S. (2013). Percepción de los estudiantes de Odontología de las estrategias metodológicas más satisfactorias utilizadas por sus académicos. Revista de Educación en Ciencias de la Salud, 10(1), 42-46. 